\title{
Turning Wood Autohydrolysate Directly into Food Packing Composite Films with Good Toughness
}

\author{
YaJie Hu, YaShuai Niu, GenQue Fu, PanPan Yue, MingFei Li, \\ Feng Peng $\mathbb{D}$, and RunCang Sun \\ Beijing Key Laboratory of Lignocellulosic Chemistry, Beijing Forestry University, Beijing 100083, China \\ Correspondence should be addressed to Feng Peng; fengpeng@bjfu.edu.cn
}

Received 29 November 2017; Revised 6 February 2018; Accepted 19 February 2018; Published 25 March 2018

Academic Editor: Jingsan Xu

Copyright (c) 2018 Yajie Hu et al. This is an open access article distributed under the Creative Commons Attribution License, which permits unrestricted use, distribution, and reproduction in any medium, provided the original work is properly cited.

\begin{abstract}
Bio-based composite films were produced by incorporating wood autohydrolysate (WH), chitosan (CS), and cellulose nanocrystals (CNC). In this work, WH was directly utilized without further purification, and CNC was introduced as the reinforced material to prepare WH-CS-CNC composite films with excellent properties. The effects of CNC on the properties of WH-CS-CNC composite films were investigated by characterizing their structures, mechanical properties, oxygen barrier, and thermal stability properties. The results suggested that $\mathrm{CNC}$ could improve tensile strength of the composite films, and the tensile strain at break could be up to $4.7 \%$. Besides, the oxygen permeability of the prepared composite films could be as low as $3.57 \mathrm{~cm}^{3} / \mathrm{day} \cdot \mathrm{m}^{2} \cdot \mathrm{kPa}$, making them suitable for the food packaging materials. These above results showed that the addition of CNC is an effective method to enhance the toughness of composite films. In addition, WH-CS-CNC composite films have great potential in the field of sustainable food packing materials.
\end{abstract}

\section{Introduction}

Film materials play an irreplaceable role in the packaging field, and they have brought great convenience to our life. Traditional oil-based polymer film materials are difficult to degrade, white pollution of which brings great harm to our earth [1]. Therefore, special attention has been given to renewable and bio-based materials because of their abundance, biodegradability, biocompatibility, and low toxicity [2-5]. Besides, bio-based materials (chitosan [6], starch [7], hemicelluloses $[6,8]$, and so on) are suitable for food packaging film due to their excellent oxygen barrier. As we know, high mechanical strength, flexibility, and good oxygen barriers are essential for the food packaging film [9].

Wood autohydrolysate $(\mathrm{WH})$ is the liquid obtained by hydrothermal extraction of lignocellulosic biomass, which is widely available in the pulping industry [10-13]. Traditionally, the hydrolysate was removed in the pulping process and did not receive sufficient attention. Thus, the extraction and utilization of WH are extremely urgent. Hydrothermal treatment is the most commonly used extraction method [14-16], during which high pressure hot water is used to deal with lignocellulosic biomass, thus causing the extraction and dissolution of hemicelluloses and the generation of low-molecular weight polymers. It is well known that the major components of $\mathrm{WH}$ are considerable amounts of hemicelluloses, oligosaccharides, and slight lignin [17]. Excellent gas barriers of hemicelluloses make them suitable for coatings and films in the food packaging field [18-20]. However, two obvious drawbacks of hemicelluloses need to be overcome, that is, the typical brittleness $[10,21]$ and the inherent hydrophilicity $[10,22]$. Several methods are commonly used to improve the performance of hemicelluloses films, including adding a certain amount of plasticizers (sorbitol, glycerol, and xylitol) in hemicelluloses solution [23, 24], in the presence of plasticizers, mixing hemicelluloses and other natural polymers (starch, chitosan, cellulose nanofibers, lignin, carboxymethyl cellulose, and sodium alginate) [3, 22], and chemical modification of hemicelluloses (esterification, etherification, cross-linking, and graft copolymerization) $[25,26]$. Although $\mathrm{WH}$ has great potential to convert into materials and fuels, the utilization of WH has not been widely studied. Therefore, more efforts could be focused on the utilization of $\mathrm{WH}$, especially in food packaging field. 
Chitosan (CS) is the $N$-deacetylated derivative of chitin, with the primary amino at the C-2 position of the $D$ glucosamine residues, which is linear natural polysaccharide. The free amino and hydroxyl in the structure tend to form secondary interactions with other polymers via hydrogen bonding or chemical cross-linking, thus improving the solubility and allowing specific chemical reactions. Because of the antimicrobial activity, nontoxicity, biodegradability, and biocompatibility of chitosan [7, 27], it has a great potential in bio-application, such as blood coagulation and wound healing, drug release and delivery, and tissue engineering [28, 29]. It is a good precursor to process into various materials, such as films, hydrogels, and nanoparticles. In addition, chitosan has a wide range of applications in food industry due to the effect of keeping meat and fruits fresh and having antimicrobial and antioxidant effects. Cellulose nanocrystals (CNC), also known as nanocellulose, have high purity, high crystallinity, high Young's modulus, and high strength, as well as the biodegradability, biocompatibility, and reproducibility. CNC can improve the thermal stability, mechanical strength, and flexibility of the composites [30,31], thus showing a wide application prospect in the preparation of high performance composites.

WH-CS composite films were prepared in our previous study, and the composite films exhibited good mechanical performance and oxygen barrier properties. However, the toughness of the composite films was extremely poor. To meet the challenge, the goal of this work is to improve the toughness of composite films by adding CNC. As reinforced filler, the high aspect ratio and good mechanical strength of CNC play a critical role. CNC could form interactions (strong hydrogen bonding and van der Waals forces) with hemicelluloses in WH [32], thus weakening chemical bonds between $\mathrm{WH}$ and $\mathrm{CS}$, softening the rigid structure of the composite films, and enhancing the toughness of composite films. On the basis of WH-CS composite films, the WHCS-CNC composite films with different CNC contents were prepared. Besides, the $\mathrm{WH}$ in our work was directly utilized without further purification. Here, we investigated the effect of $\mathrm{CNC}$ on the properties of the WH-based films and revealed relationship between the structure and properties of nanocomposite films.

\section{Experimental}

2.1. Materials. The wood autohydrolysate (WH) is the liquid obtained by hydrothermal treatment of poplar wood chips, provided by Shandong Sun Paper Industry Joint Stock Co., Ltd. WH accounts for $98.35 \%$ of total weight of the liquid, which has an average molecular weight $(M w)$ of about $2300 \mathrm{~g} / \mathrm{mol}$ and a polydispersity index value (PDI) of 8.80. The components of wood autohydrolysate $(\mathrm{WH})$ were mainly $61.3 \%$ hemicelluloses, $10.5 \%$ lignin, $12.8 \%$ monosaccharide, $13.7 \%$ oligosaccharide, and $1.65 \%$ other insoluble materials of dry WH. The main chemical structure of the hemicelluloses in $\mathrm{WH}$ is $\mathrm{O}$-acetyl-4-O-methylglucuronoxylan. Compared with hemicelluloses extracted by alkali treatment, the hemicelluloses in WH exhibit lower molecular weight and more
TABLE 1: The composite films prepared by different volume ratios of $\mathrm{WH}, \mathrm{CS}$, and CNC.

\begin{tabular}{lc}
\hline Sample name & Proportion \\
\hline $\mathrm{F}_{0.01}$ & $V(\mathrm{WH}) / V(\mathrm{CS}) / V(\mathrm{CNC})=1: 1: 0.01$ \\
$\mathrm{~F}_{0.03}$ & $V(\mathrm{WH}) / V(\mathrm{CS}) / V(\mathrm{CNC})=1: 1: 0.03$ \\
$\mathrm{~F}_{0.05}$ & $V(\mathrm{WH}) / V(\mathrm{CS}) / V(\mathrm{CNC})=1: 1: 0.05$ \\
$\mathrm{~F}_{0.08}$ & $V(\mathrm{WH}) / V(\mathrm{CS}) / V(\mathrm{CNC})=1: 1: 0.08$ \\
$\mathrm{~F}_{0.1}$ & $V(\mathrm{WH}) / V(\mathrm{CS}) / V(\mathrm{CNC})=1: 1: 0.1$ \\
\hline
\end{tabular}

excellent solubility, which is beneficial for further conversion. Chitosan, with degree of acetylation of $80-95 \%$, molecular weight of $200,000 \mathrm{~g} / \mathrm{mol}$, and viscosity of $50-800 \mathrm{mPa} \cdot \mathrm{s}$, was provided by Sinopharm Chemical Reagent Co., Ltd. (Beijing). Cellulose nanocrystals (CNC) were prepared by acidification of microcrystalline cellulose. The microcrystalline cellulose was provided by the Sinopharm Chemical Reagent Co., Ltd. (Beijing), with the polymerization degree of 210-240. All reagents mentioned above were directly used without further purification.

2.2. Preparation of CNC. Cellulose nanocrystals (CNC) are the reinforced materials during the preparation of composite films. CNC were prepared by the sulfuric acid hydrolysis of microcrystalline cellulose, and microcrystalline cellulose was treated with $60 \%$ concentrated sulfuric acid during $30 \mathrm{~min}$ at $45^{\circ} \mathrm{C}$. After acidification, excessive deionized water was added to the mixture to stop the reaction, and the reaction mixtures were allowed to stand for $24 \mathrm{~h}$. Then, the supernatant was discarded and the remaining sediment was poured into a dialysis bag to dialyze for approximately one week until the $\mathrm{pH}$ of the dialysate was neutral. Dialysis products (CNC) were characterized by AFM and the solid contents were determined by heating with high temperature in muffle furnace.

\subsection{Preparation of Composite Films. The WH-CS-CNC} nanocomposite films were prepared by a typical method, mixing the wood hydrolysate (WH), chitosan (CS), and cellulose nanocrystals (CNC), and casting. Firstly, the solutions of each component were prepared, respectively. The $\mathrm{WH}$ was diluted to $3 \%(\mathrm{wt})$ solution under vigorous stirring. $1 \%$ acetic acid and deionized water were added to CS and stirred at $60^{\circ} \mathrm{C}$ for $5 \mathrm{~h}$ to prepare $3 \%(\mathrm{wt})$ chitosan solution. The premade CNC were also formulated to $3 \%(\mathrm{wt})$ solution, and CNC solution was stirred well before being used to avoid agglomeration. Based on our previous study on WH-CS film, the optimal proportion of WH and CS is $1: 1$; here CNC were added to the mixture of $\mathrm{WH}$ and $\mathrm{CS}$ with different ratios and then stirred vigorously at $60^{\circ} \mathrm{C}$ until uniform solutions. Finally, the mixed solutions $(10 \mathrm{ml})$ were poured into plastic Petri dish and left to dry at $50^{\circ} \mathrm{C}$ in vacuum oven. The films were formulated and samples were labeled as shown in Table 1 .

2.4. Characterization of Composite Films. The CNC were characterized by a multimode 8 atomic force microscopy (Bruker, Germany). Images were obtained in phase contrast 
mode using a monolithic silicon tip with a resonance frequency of $300 \mathrm{kHz}$ and a scan angle of $0^{\circ}$. The FT-IR spectra of composite films were recorded on a Nicolet iN 10 FTIR Spectrometer (Thermo Nicolet Corporation, Madison, WI) using thermally treated $\mathrm{KBr}$ pellets, and the spectra were recorded from 4000 to $500 \mathrm{~cm}^{-1}$. A Hitachi S-3400N II (Hitachi, Japan) instrument was used to capture the pictures of samples at $15 \mathrm{kV}$. The composite film samples were firstly quenched by liquid nitrogen and then sputter-coated with a thin layer of gold. The surface morphology and crosssectional structure of composite films were observed at magnifications ranging from $2000 x$ to $3000 x$.

2.5. Tensile Strength Testing. The tensile strength tests of composite films were carried out by universal tensile testing machine (UTM6503, Shenzhen Suns Technology Stock Co. Ltd., China). The films were cut into $40 \mathrm{~mm} \times 10 \mathrm{~mm}$ rectangular specimens with the premeasured thickness using a paper thickness gauge (ZH-4, Changchun Paper Testing Machine Co. Ltd., China); then rectangular specimens were tested with strain rate of $5 \mathrm{~mm} / \mathrm{min}$ at room temperature. For each composite film, at least 5 individual specimens were tested.

2.6. Thermal Behavior Analysis. Thermal properties of composite films were analyzed by thermogravimetric analysis (TGA) and derivative thermogravimetry (DTG) on a simultaneous thermal analyzer (DTG-60, Shimadzu, Japan), with the heating temperature ranging from $30^{\circ} \mathrm{C}$ to $700^{\circ} \mathrm{C}$ and the heating rate of $10^{\circ} \mathrm{C} / \mathrm{min}$.

2.7. Oxygen Permeability. The oxygen permeability (OP) of WH-based composite films was determined with a VACV1 permeability analyzer. The composite films were cut and sealed between aluminum foils with the effective exposed area of $5.0 \mathrm{~cm}^{2}$. Samples were stored at the following conditions for one week: temperature is $23^{\circ} \mathrm{C}$ and the relative humidity (RH) is $0 \%$. At least two individual samples were measured for each composite film.

\section{Results and Discussion}

3.1. Characterization of CNC. CNC were prepared from microcrystalline cellulose by acidification with $60 \%$ concentrated sulfuric acid. The AFM topography image of the prepared CNC is shown in Figure 1. The length of the target product is $20-140 \mathrm{~nm}$ and the diameter is in the range of $10-40 \mathrm{~nm}$. It has been reported that the $\mathrm{CNC}$ is the rigid rod-shaped cellulose with the length ranging from $10 \mathrm{~nm}$ to $1000 \mathrm{~nm}$ [33]. The size of the prepared product is in accordance with the literature results. Therefore, the product prepared was verified to $\mathrm{CNC}$.

3.2. The WH-CS-CNC Composite Films. Composite films were prepared by chemical cross-linking of WAH, CS, and $\mathrm{CNC}$. The photos of the WH-CS film without $\mathrm{CNC}\left(\mathrm{F}_{0}\right)$ and the film $\mathrm{F}_{0.08}$ are shown in Figure 2. As can be seen, the two composite films both exhibit good transmittance. Compared

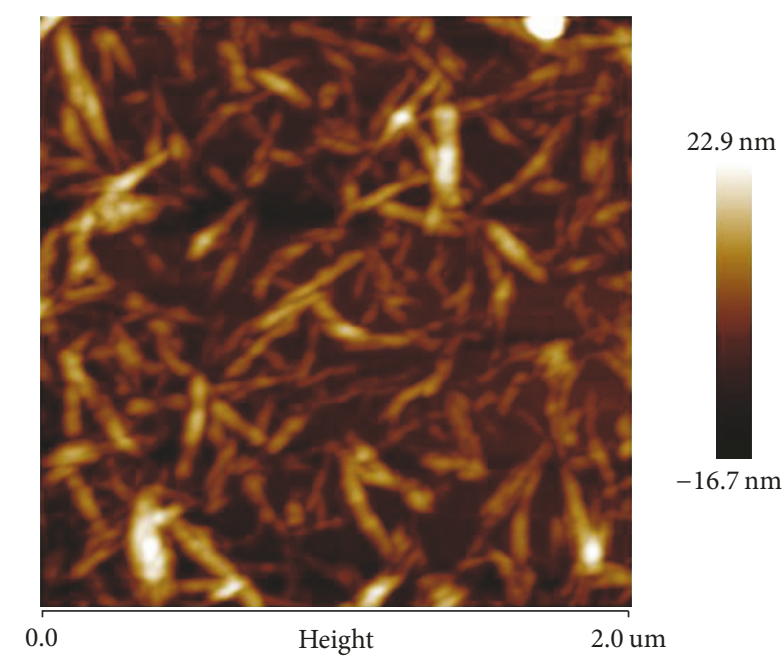

Figure 1: The AFM image of CNC.

with the film $\mathrm{F}_{0}, \mathrm{~F}_{0.08}$ has a smoother surface, which indicates that the addition of CNC makes a more homogeneous solution. The smooth and uniform surface of WAH-CSCNC composite films has a positive effect on the application in the field of food packaging materials.

3.3. Structure of WH-CS-CNC Composite Films. The structure-property relationship and cross-linking mechanism of the WH-CS-CNC composite films were verified in the FTIR spectra. The FT-IR spectra of raw materials ( $\mathrm{WH}, \mathrm{CS}$, and $\mathrm{CNC}$ ) and WH-CS-CNC composite films with different $\mathrm{CNC}$ contents are shown in Figure 3, which reveal the formation mechanism of composite films. As shown in Figure 3(a), the characteristic peak of $\mathrm{WH}$ is presented at $1731 \mathrm{~cm}^{-1}$ originating from $\mathrm{C}=\mathrm{O}$ group, and the signal at $1035 \mathrm{~cm}^{-1}$ is the typical C-O-C stretching of glycosidic linkages. In the spectrum of CS, the major peak appearing at $1650 \mathrm{~cm}^{-1}$ is assigned to the $-\mathrm{NH}$ stretching vibration and the absorption band at $1395 \mathrm{~cm}^{-1}$ is attributed to the $-\mathrm{NH}_{2}$. As shown in Figure 3(b), FT-IR spectra of different composite films $\mathrm{F}_{0}$, $\mathrm{F}_{0.03}$, and $\mathrm{F}_{0.08}$ present the same absorption peaks, and the intensity of absorption peaks increases with the increment of CNC contents. In addition, the new peaks at $1716 \mathrm{~cm}^{-1}$ and $1559 \mathrm{~cm}^{-1}$ originating from the stretching vibration of $\mathrm{C}=\mathrm{N}$ are present in the spectra of composite films, which substitute the original peak at $1731 \mathrm{~cm}^{-1}$ in $\mathrm{WH}$ spectrum and characteristic peak at $1650 \mathrm{~cm}^{-1}$ in CS spectrum. The emergence of new peaks confirmed that the Maillard reaction occurred during the preparation progress of the composite films; that is to say, hemicellulose aldehyde $(\mathrm{C}=\mathrm{O})$ in $\mathrm{WH}$ was reacted with the amino $\left(-\mathrm{NH}_{2}\right)$ in CS, producing Schiff base $(\mathrm{C}=\mathrm{N})$.

3.4. Morphology of WH-CS-CNC Composite Films. The surface morphology and cross-sectional structure of composite films $\mathrm{F}_{0}, \mathrm{~F}_{0.03}$, and $\mathrm{F}_{0.08}$ were investigated by SEM, and the images are presented in Figure 4. Figures 4(a), 4(c), and 4(e) show that composite films $\mathrm{F}_{0}, \mathrm{~F}_{0.03}$, and $\mathrm{F}_{0.08}$ have a 

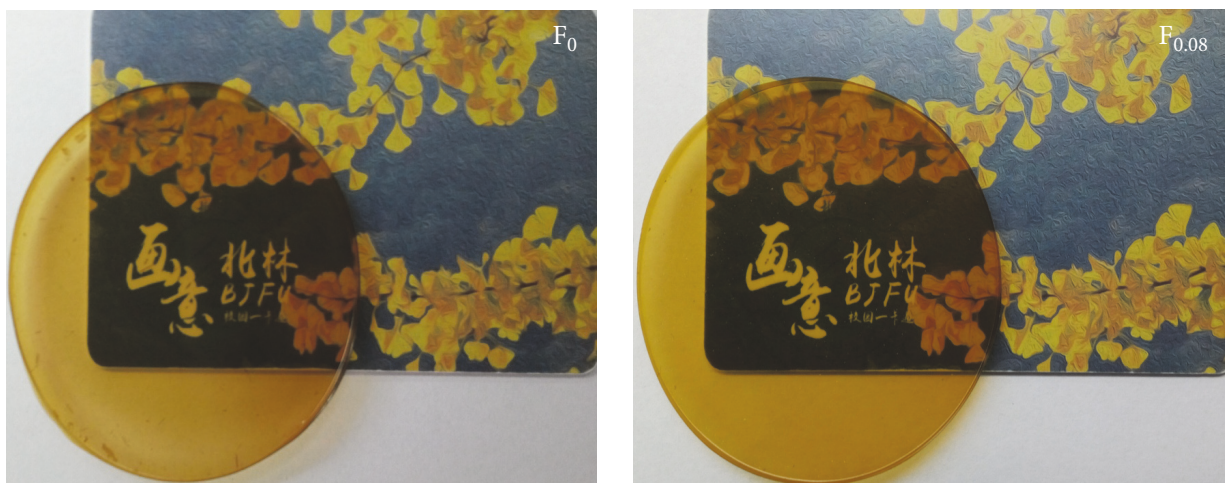

Figure 2: The photos of composite films $\mathrm{F}_{0}$ and $\mathrm{F}_{0.08}$.

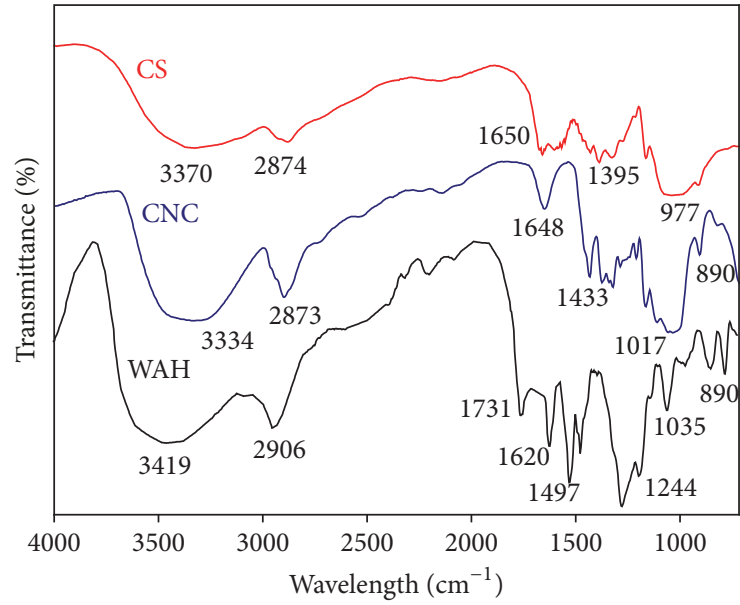

(a)

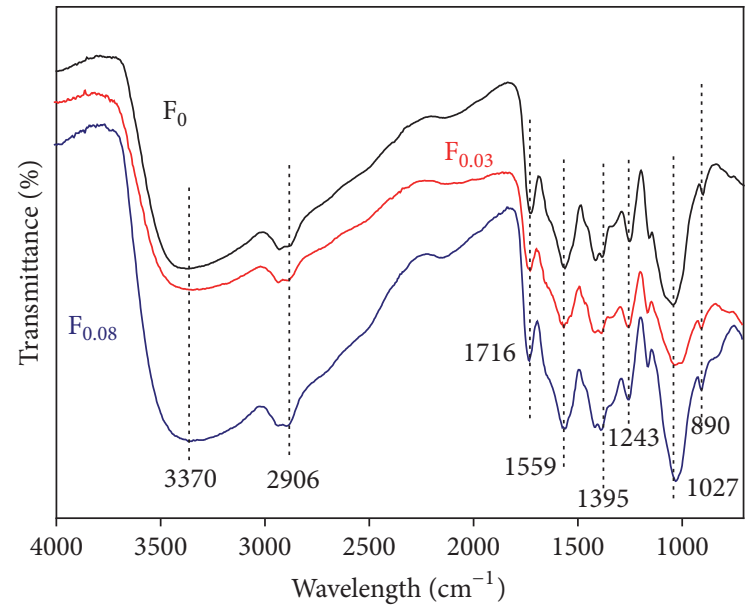

(b)

Figure 3: (a) The FT-IR spectra of WH, CS, and CNC. (b) The FT-IR spectra of the composite films $\left(\mathrm{F}_{0}, \mathrm{~F}_{0.03}\right.$, and $\left.\mathrm{F}_{0.08}\right)$.

highly aligned structure, and all present the smooth surface. However, the images of the cross-section are slightly different, as shown in Figures 4(b), 4(d), and 4(f). The cross-sectional structure of film $\mathrm{F}_{0}$ is tighter and rougher than $\mathrm{F}_{0.03}$ and $\mathrm{F}_{0.08}$, whereas the cross-sectional structures of the composite films $\mathrm{F}_{0.03}$ and $\mathrm{F}_{0.08}$ have more laminar morphology. The reason of the more laminar structure of composite films could be ascribed to the addition of $\mathrm{CNC}$; $\mathrm{CNC}$ can form interactions (strong hydrogen bonding and van der Waals forces) with hemicelluloses in WH, weaken chemical bonds between WH and CS, and soften the rigid structure of the composite films. The smooth surface of WH-CS-CNC composite films makes them more suitable for packaging materials.

3.5. Thermal Behavior Analysis. The thermal performance of the WH-CS-CNC composite films was analyzed by thermogravimetric analysis (TGA). The thermogravimetric curve and the derivative thermogravimetric curve (DTG) of the composite films are presented in Figure 5. As can be seen from Figure 5(a), the thermogravimetric curves of composite films $\mathrm{F}_{0}, \mathrm{~F}_{0.03}$, and $\mathrm{F}_{0.08}$ are similar. The solid residues at $700^{\circ} \mathrm{C}$ of composite films $\mathrm{F}_{0}, \mathrm{~F}_{0.03}$, and $\mathrm{F}_{0.08}$ are $34.0 \%, 36.3 \%$,
TABLE 2: Thermal property of TGA curves of composite films $\left(\mathrm{F}_{0}\right.$, $\mathrm{F}_{0.03}$, and $\left.\mathrm{F}_{0.08}\right)$.

\begin{tabular}{lccc}
\hline Curve & $\mathrm{F}_{0}$ & $\mathrm{~F}_{0.03}$ & $\mathrm{~F}_{0.08}$ \\
\hline$T_{\text {onset }}\left({ }^{\circ} \mathrm{C}\right)$ & 132 & 134 & 138 \\
$T_{\max }\left({ }^{\circ} \mathrm{C}\right)$ & 267 & 270 & 273 \\
Residuals $(w t \%)$ at $700^{\circ} \mathrm{C}$ & 34.0 & 36.3 & 37.5 \\
\hline
\end{tabular}

and $37.5 \%$, respectively, which implies the incorporation of CNC resulted in better thermal stability. From Figure 5(b), the mass loss of WH-CS-CNC composite films in the temperature of $100-150^{\circ} \mathrm{C}$ relating to the evaporation of water is slight. In the range of $200-700^{\circ} \mathrm{C}$, mass loss of the composite films reached the maximum value, which was mainly due to the degradation of $\mathrm{WH}, \mathrm{CS}$, and CNC. In addition, the initial decomposition temperature $\left(T_{\text {onset }}\right)$ and the temperature of maximum mass loss $\left(T_{\max }\right)$ of DTG curve are shown in Table 2. Obviously, $T_{\text {onset }}, T_{\max }$, and residuals of composite films increase with the increment of $\mathrm{CNC}$, indicating that $\mathrm{CNC}$ has positive effect on the thermal performance of composite films, and the addition of CNC improves the thermal stability of the composite films. 




(a)

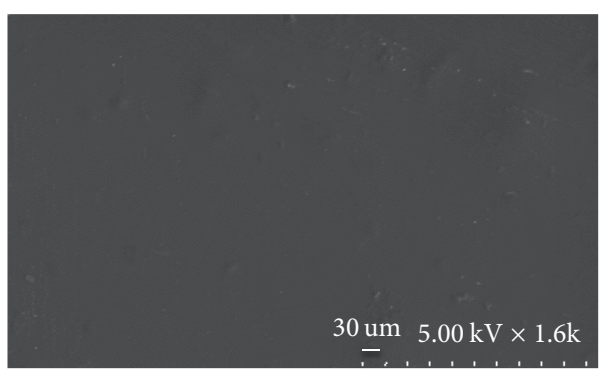

(c)

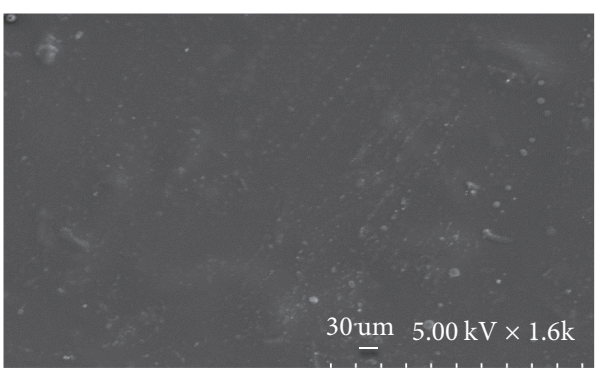

(e)

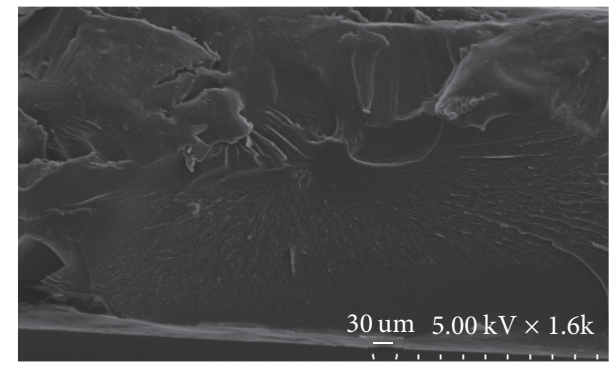

(b)

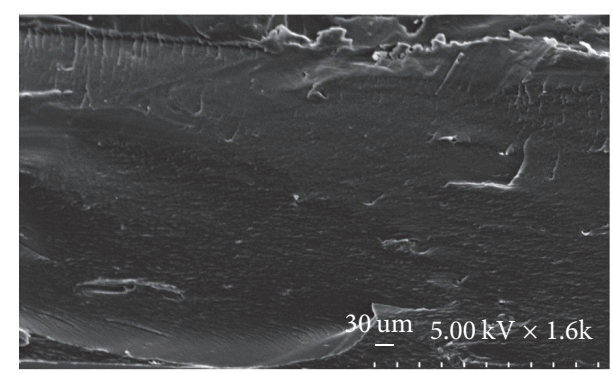

(d)

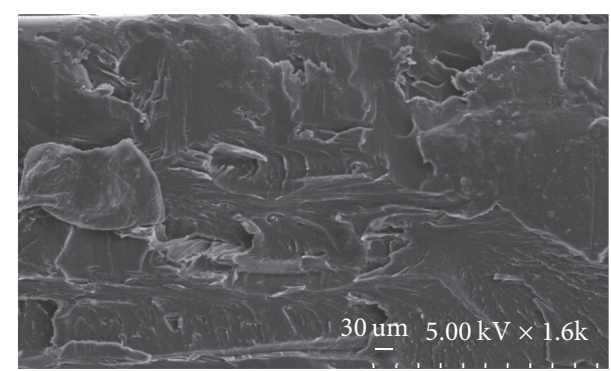

(f)

Figure 4: The SEM images of composite films $\mathrm{F}_{0}(\mathrm{a}, \mathrm{b}), \mathrm{F}_{0.03}(\mathrm{c}, \mathrm{d})$, and $\mathrm{F}_{0.08}(\mathrm{e}, \mathrm{f})$.

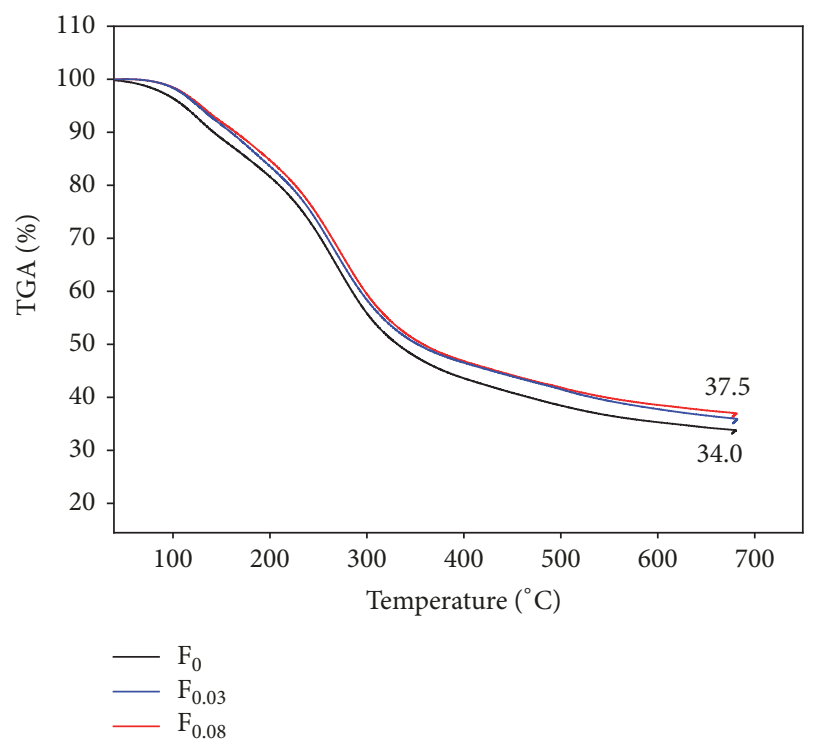

(a)

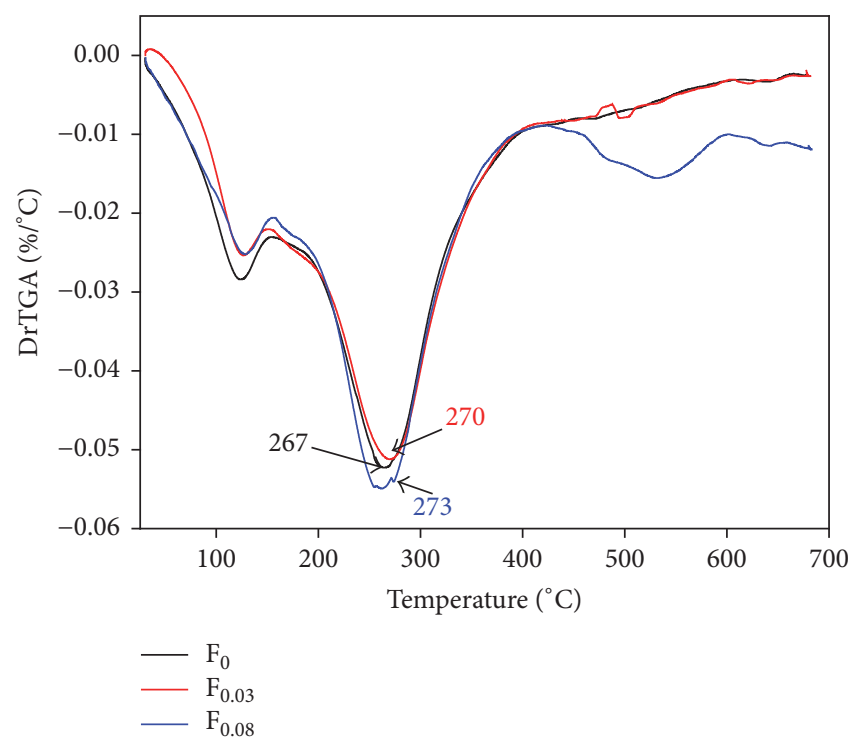

(b)

Figure 5: (a) The TGA curves of composite films $\left(\mathrm{F}_{0}, \mathrm{~F}_{0.03}\right.$, and $\left.\mathrm{F}_{0.08}\right)$. (b) The DTG curves of composite films $\left(\mathrm{F}_{0}, \mathrm{~F}_{0.03}\right.$, and $\left.\mathrm{F}_{0.08}\right)$. 


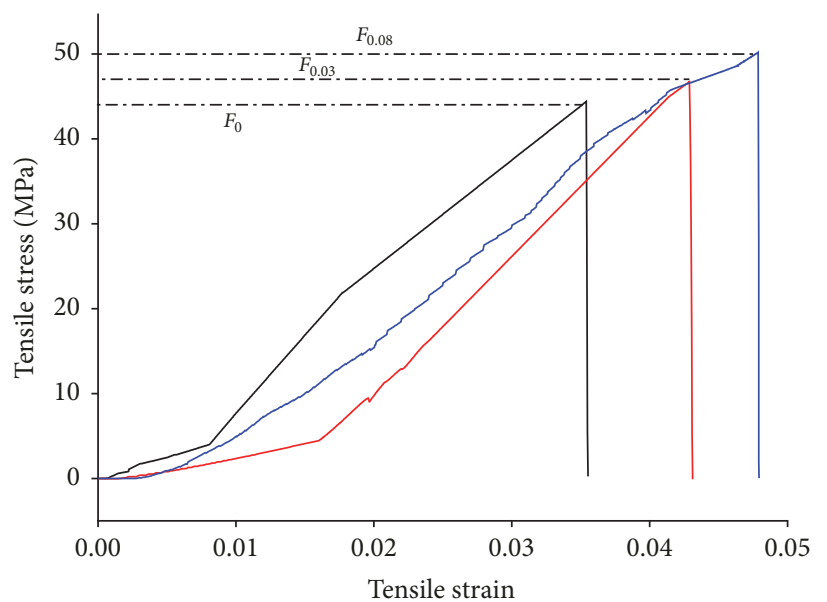

(a)

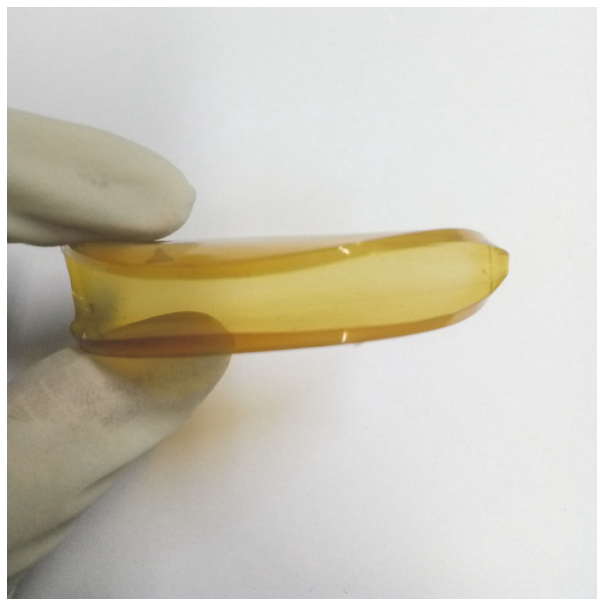

(b-1)

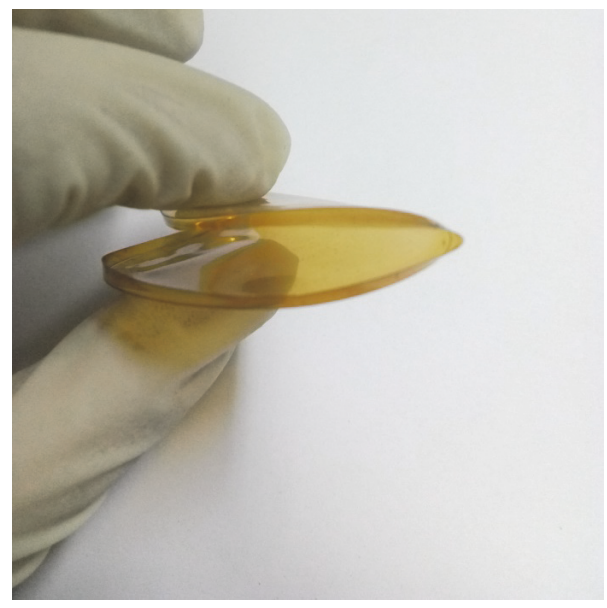

(b-2)

(b)

Figure 6: (a) Tensile-strain curves of composite films $\left(\mathrm{F}_{0}, \mathrm{~F}_{0.03}\right.$, and $\left.\mathrm{F}_{0.08}\right)$. (b) Toughness tests of composite film $\mathrm{F}_{0.08}$

3.6. Mechanical Property of WH-CS-CNC Composite Films. Adequate mechanical property is important for the packing materials, and CNC is an excellent reinforced filler with high aspect ratio and good mechanical strength; thus the addition of CNC could effectively improve the mechanical performance of composite films. The tensile-strain curves of composite films $\mathrm{F}_{0}, \mathrm{~F}_{0.03}$, and $\mathrm{F}_{0.08}$ are shown in Figure 6(a). As shown in Figure 6(a), the tensile strengths of composite films $\mathrm{F}_{0}, \mathrm{~F}_{0.03}$, and $\mathrm{F}_{0.08}$ are $44.3,46.5$, and $49.1 \mathrm{MPa}$, respectively, indicating that $\mathrm{CNC}$ result in significant improvement in the tensile strength of composite films. Besides, the values of tensile strain at break recorded in Table 3 show that the tensile strain at break increases with the increment of CNC. Figure 6(b) presents the results of toughness tests on composite films $\mathrm{F}_{0.08}$, showing that excellent toughness is in accordance with the good tensile strain at break. CNC improved the toughness of the composite films to some extent, because CNC could interact with the molecular chains of hemicelluloses, then the intermolecular interaction between WH and CS was weakened, and the rigid structure of composite films was softened. These results reveal that the
TABle 3: Tensile testing results of composite films $\left(\mathrm{F}_{0}, \mathrm{~F}_{0.03}\right.$, and $\left.\mathrm{F}_{0.08}\right)$.

\begin{tabular}{lccc}
\hline Sample & $\begin{array}{c}\text { Tensile } \\
\text { strength } \\
(\mathrm{MPa})\end{array}$ & $\begin{array}{c}\text { Tensile strain at } \\
\text { break }(\%)\end{array}$ & Thickness $(\mu \mathrm{m})$ \\
\hline $\mathrm{F}_{0}$ & $44.3 \pm 0.5$ & $3.5 \pm 0.1$ & $77.4 \pm 3.0$ \\
$\mathrm{~F}_{0.03}$ & $46.5 \pm 1.0$ & $4.3 \pm 0.2$ & $84.6 \pm 2.0$ \\
$\mathrm{~F}_{0.08}$ & $49.1 \pm 1.5$ & $4.7 \pm 0.1$ & $88.1 \pm 3.0$ \\
\hline
\end{tabular}

WH-CS-CNC composite films not only have good tensile strength properties but also exhibit excellent toughness, which provide a greater chance for the application in food packaging materials.

3.7. Oxygen Permeability. The oxygen permeability (OP) of WH-CS-CNC composite films was determined by the differential pressure method with a VAC-V1 permeability analyzer. The results of the oxygen permeability are shown 
TABLE 4: The OP values of composite films $\left(\mathrm{F}_{0}, \mathrm{~F}_{0.03}\right.$, and $\left.\mathrm{F}_{0.08}\right)$.

\begin{tabular}{lcc}
\hline Sample & $\mathrm{OP}\left(\mathrm{cm}^{3} \cdot \mathrm{m}^{-2} \cdot 24 \mathrm{~h}^{-1} \cdot \mathrm{kPa}^{-1}\right)$ & Test area $\left(\mathrm{cm}^{2}\right)$ \\
\hline $\mathrm{F}_{0}$ & $1.54 \pm 0.04$ & 5.0 \\
$\mathrm{~F}_{0.03}$ & $3.57 \pm 0.02$ & 5.0 \\
$\mathrm{~F}_{0.08}$ & $5.14 \pm 0.05$ & 5.0 \\
\hline
\end{tabular}

in Table 4. As shown in Table 4, all composite films exhibit remarkably good oxygen barriers with the OP values below $1.54,3.57$, and $5.14 \mathrm{~cm}^{3} \cdot \mathrm{m}^{-2} \cdot 24 \mathrm{~h}^{-1} \cdot \mathrm{kPa}^{-1}$, respectively. When $\mathrm{CNC}$ was added to the WH-CS mixture, CNC weakened chemical bonds between WH and CS and softened the rigid structure of the composite films, thus increasing the oxygen permeability of the film. The maximum $O P$ value of $10 \mathrm{~cm}^{3} \cdot \mathrm{m}^{-2} \cdot 24 \mathrm{~h}^{-1} \cdot \mathrm{kPa}^{-1}$ or below is considered to be the standard for food packaging materials [34]; therefore, all prepared WH-CS-CNC composite films meet the standard for food packaging materials.

\section{Conclusions}

In this work, the WH-CS-CNC composite films were prepared with different $\mathrm{CNC}$ contents. Compared with $\mathrm{WH}-$ CS composite film $\mathrm{F}_{0}$, WH-CS-CNC composite films have better toughness. Because CNC, as reinforced filler, interacts with the molecular chains of hemicelluloses, thus it weakens chemical bonds between WH and CS and softens the rigid structure of composite films. It is also noteworthy that the addition of CNC improved the mechanical performance and thermal stability of the films to some extent. Although the oxygen barrier of WH-CS-CNC composite films was slightly poorer than WH-CS composite films, the OP values of $\mathrm{WH}$ CS-CNC composite films still coincided with the standard $\mathrm{OP}$ of food packing materials. These results indicated that the WH-CS-CNC composite films may have great potential in the field of packaging materials from the economic and environmental perspective.

\section{Conflicts of Interest}

The authors declare that they have no conflicts of interest.

\section{Acknowledgments}

This work was supported by the Fundamental Research Funds for Central Universities (JC2015-03), Beijing Municipal Natural Science Foundation (6182031), Author of National Excellent Doctoral Dissertations of China (201458), and the National Program for Support of Top-Notch Young Professionals.

\section{References}

[1] A. Höije, M. Gröndahl, K. Tømmeraas, and P. Gatenholm, "Isolation and characterization of physicochemical and material properties of arabinoxylans from barley husks," Carbohydrate Polymers, vol. 61, no. 3, pp. 266-275, 2005.
[2] Y. Lv and Z. Ma, "Research status and application of biodegradable packaging film materials," Journal of Packaging Preface, vol. 4, pp. 32-33, 2016.

[3] U. Edlund, Y. Z. Ryberg, and A.-C. Albertsson, "Barrier films from renewable forestry waste," Biomacromolecules, vol. 11, no. 9, pp. 2532-2538, 2010.

[4] Y. Z. Zhu Ryberg, U. Edlund, and A.-C. Albertsson, "Retrostructural model to predict biomass formulations for barrier performance," Biomacromolecules, vol. 13, no. 8, pp. 2570-2577, 2012.

[5] W. Boonkong, A. Petsom, and N. Thongchul, "Rapidly stopping hemorrhage by enhancing blood clotting at an opened wound using chitosan/polylactic acid/polycaprolactone wound dressing device," Journal of Materials Science: Materials in Medicine, vol. 24, no. 6, pp. 1581-1593, 2013.

[6] Y. Guan, X.-M. Qi, G.-G. Chen, F. Peng, and R.-C. Sun, "Facile approach to prepare drug-loading film from hemicelluloses and chitosan," Carbohydrate Polymers, vol. 153, pp. 542-548, 2016.

[7] M. Avella, J. J. De Vlieger, M. E. Errico, S. Fischer, P. Vacca, and M. G. Volpe, "Biodegradable starch/clay nanocomposite films for food packaging applications," Food Chemistry, vol. 93, no. 3, pp. 467-474, 2005.

[8] G.-G. Chen, X.-M. Qi, M.-P. Li et al., "Hemicelluloses/montmorillonite hybrid films with improved mechanical and barrier properties," Scientific Reports, vol. 5, Article ID 16405, 2015.

[9] V. Siracusa, P. Rocculi, S. Romani, and M. D. Rosa, "Biodegradable polymers for food packaging: a review," Trends in Food Science \& Technology, vol. 19, no. 12, pp. 634-643, 2008.

[10] S. Saadatmand, U. Edlund, A.-C. Albertsson, S. Danielsson, O. Dahlman, and K. Karlström, "Turning hardwood dissolving pulp polysaccharide residual material into barrier packaging," Biomacromolecules, vol. 14, no. 8, pp. 2929-2936, 2013.

[11] J. Thornton, R. Ekman, B. Holmbom, and F. ÖRså, "Polysaccharides dissolved from norway spruce in thermomechanical pulping and peroxide bleaching," Journal of Wood Chemistry and Technology, vol. 14, no. 2, pp. 159-175, 1994.

[12] H. Li, A. Saeed, M. S. Jahan, Y. Ni, and A. Van Heiningen, "Hemicellulose removal from hardwood chips in the prehydrolysis step of the kraft-based dissolving pulp production process," Journal of Wood Chemistry and Technology, vol. 30, no. 1, pp. 48-60, 2010.

[13] A. Ibn Yaich, U. Edlund, and A.-C. Albertsson, "Wood hydrolysate barriers: performance controlled via selective recovery," Biomacromolecules, vol. 13, no. 2, pp. 466-473, 2012.

[14] O. Bobleter, "Hydrothermal degradation of polymers derived from plants," Progress in Polymer Science, vol. 19, no. 5, pp. 797841, 1994.

[15] P. Kilpeläinen, K. Leppänen, P. Spetz et al., "Pressurised hot water extraction of acetylated xylan from birch sawdust," Nordic Pulp \& Paper Research Journal, vol. 27, no. 4, pp. 680-688, 2012.

[16] S.-H. Yoon and A. Van Heiningen, "Kraft pulping and papermaking properties of hot-water pre-extracted loblolly pine in an integrated forest products biorefinery," TAPPI Journal, vol. 7, no. 7, pp. 22-27, 2008.

[17] M. Lawoko, G. Henriksson, and G. Gellerstedt, "Structural differences between the lignin-carbohydrate complexes present in wood and in chemical pulps," Biomacromolecules, vol. 6, no. 6, pp. 3467-3473, 2005.

[18] R. Sun, X. F. Sun, and J. Tomkinson, "Hemicelluloses and their derivatives," ACS Symposium Series, vol. 864, pp. 2-22, 2004. 
[19] M. S. Lindblad, E. Ranucci, and A.-C. Albertsson, "Biodegradable polymers from renewable sources. New hemicellulosebased hydrogels," Macromolecular Rapid Communications, vol. 22, no. 12, pp. 962-967, 2001.

[20] K. S. Mikkonen, S. Heikkinen, A. Soovre et al., "Films from oat spelt arabinoxylan plasticized with glycerol and sorbitol," Journal of Applied Polymer Science, vol. 114, no. 1, pp. 457-466, 2009.

[21] Y. Zhu Ryberg, U. Edlund, and A.-C. Albertsson, "Innovative approaches for converting a wood hydrolysate to high-quality barrier coatings," ACS Applied Materials \& Interfaces, vol. 5, no. 16, pp. 7748-7757, 2013.

[22] A. I. Yaich, U. Edlund, and A.-C. Albertsson, "Adapting wood hydrolysate barriers to high humidity conditions," Carbohydrate Polymers, vol. 100, pp. 135-142, 2014.

[23] M. Gröndahl, L. Eriksson, and P. Gatenholm, "Material properties of plasticized hardwood xylans for potential application as oxygen barrier films," Biomacromolecules, vol. 5, no. 4, pp. 15281535, 2004.

[24] K. S. Mikkonen, M. I. Heikkilä, S. M. Willför, and M. Tenkanen, "Films from glyoxal-crosslinked spruce galactoglucomannans plasticized with sorbitol," International Journal of Polymer Science, vol. 2012, Article ID 482810, pp. 1-8, 2012.

[25] J. Hartman, A.-C. Albertsson, M. S. Lindblad, and J. Sjöberg, "Oxygen barrier materials from renewable sources: material properties of softwood hemicellulose-based films," Journal of Applied Polymer Science, vol. 100, no. 4, pp. 2985-2991, 2006.

[26] V. Coma, I. Sebti, P. Pardon, F. H. Pichavant, and A. Deschamps, "Film properties from crosslinking of cellulosic derivatives with a polyfunctional carboxylic acid," Carbohydrate Polymers, vol. 51, no. 3, pp. 265-271, 2003.

[27] M. Rinaudo, "Chitin and chitosan: properties and applications," Progress in Polymer Science, vol. 31, no. 7, pp. 603-632, 2006.

[28] R. Jayakumar, M. Prabaharan, S. V. Nair, and H. Tamura, "Novel chitin and chitosan nanofibers in biomedical applications," Biotechnology Advances, vol. 28, no. 1, pp. 142-150, 2010.

[29] J. H. Park, G. Saravanakumar, K. Kim, and I. C. Kwon, “Targeted delivery of low molecular drugs using chitosan and its derivatives," Advanced Drug Delivery Reviews, vol. 62, no. 1, pp. 28-41, 2010.

[30] T. H. Wegner and P. E. Jones, "Advancing cellulose-based nanotechnology," Cellulose, vol. 13, no. 2, pp. 115-118, 2006.

[31] A. Šturcová, G. R. Davies, and S. J. Eichhorn, "Elastic modulus and stress-transfer properties of tunicate cellulose whiskers," Biomacromolecules, vol. 6, no. 2, pp. 1055-1061, 2005.

[32] X.-W. Peng, J.-L. Ren, L.-X. Zhong, and R.-C. Sun, "Nanocomposite films based on xylan-rich hemicelluloses and cellulose nanofibers with enhanced mechanical properties," Biomacromolecules, vol. 12, no. 9, pp. 3321-3329, 2011.

[33] W. Li, R. Wang, and S. Liu, "Preparation of nanomaterials," Journal of Chemical Progress, vol. 22, no. 10, pp. 2060-2069, 2010.

[34] J. M. Krochta and D. E. Mulder-Johnston, Edible and Biodegradable Polymer Films: Challenges and Opportunities, Institute of Food Technologists, 1997. 


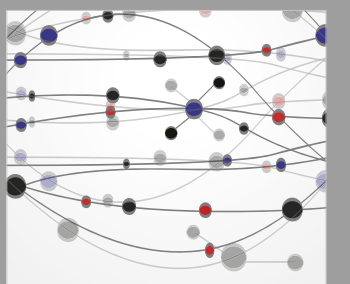

The Scientific World Journal
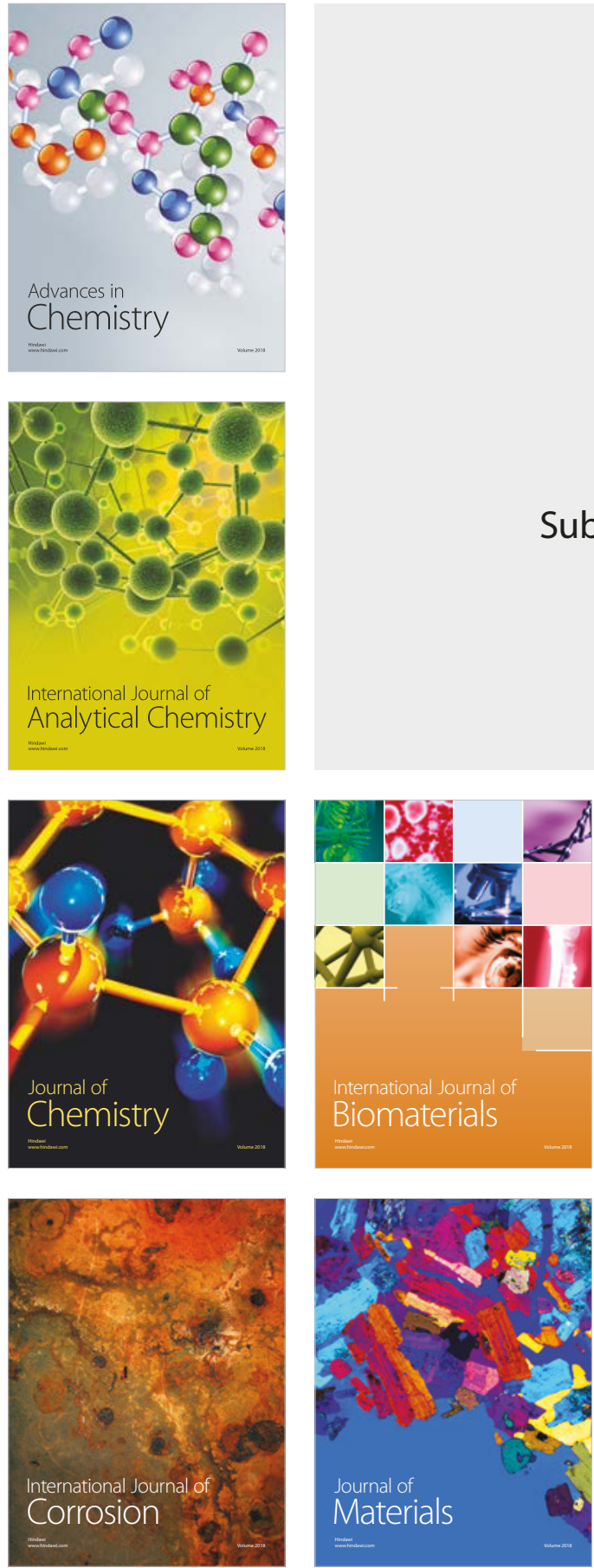

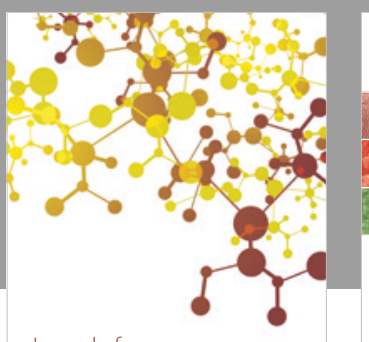

Journal of

Applied Chemistry
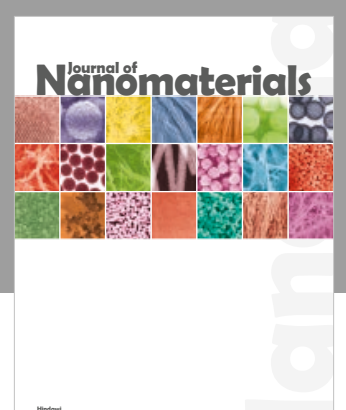

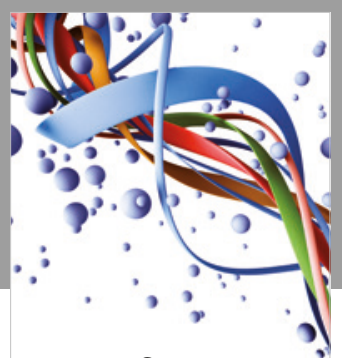

Scientifica

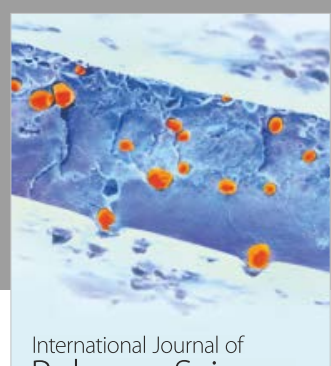

Polymer Science

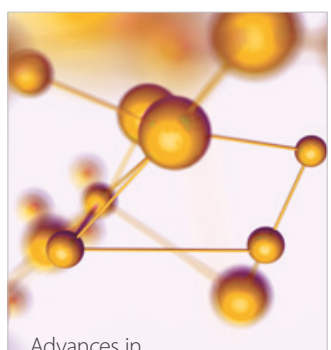

Physical Chemistry
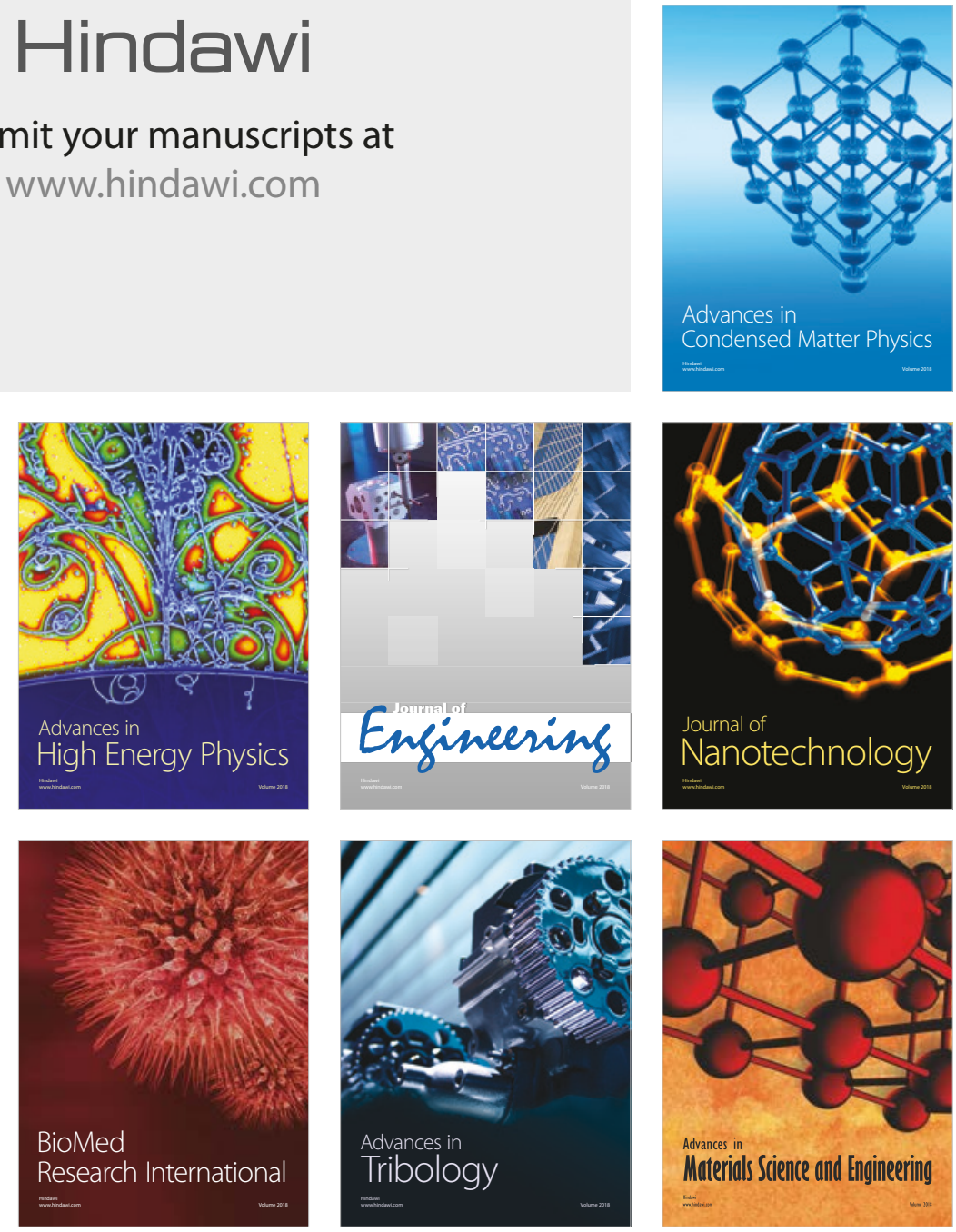